\title{
Flow visualization and skin friction determination in transitional channel flow
}

\author{
Sattaya Yimprasert ${ }^{1} \cdot$ Mathias Kvick $^{2} \cdot$ P. Henrik Alfredsson ${ }^{3} \cdot$ Masaharu Matsubara $^{1}[$
}

Received: 31 March 2020 / Revised: 12 October 2020 / Accepted: 17 November 2020 / Published online: 30 January 2021

(c) The Author(s) 2021

\begin{abstract}
The present study experimentally determines the transitional Reynolds number range for plane channel flow and characterizes its transitional state. The pressure along the channel is measured to determine the skin friction coefficient as function of Reynolds number from the laminar state, through the transitional region into the fully turbulent state. The flow structure was studied through flow visualisation which shows that as the Reynolds number increases from the laminar state the transitional region starts showing randomly occurring turbulent spots. With increasing Reynolds number the spots shift into oblique patches and bands of small scale turbulence that form across the channel width, together with large-scale streaky structures found in areas between the turbulent regions. An image analysing technique was used to determine the intermittency factor, i.e. the turbulence fraction in the flow, as function of Reynolds number. It is found that the skin friction coefficient reaches its turbulent value before the flow is fully turbulent (the intermittency factor is still below one). This suggests that the observed streaky structures in non-turbulent regions contribute to the enhancement of the wall-normal transfer of momentum. Also above the Reynolds numbers where the turbulent skin friction coefficient has been established large-scale features consisting of irregular streaky structures are found. They have an oblique shape similar to the non-turbulent and turbulent patches in the transitional flow indicating that the transition process is not fully complete even above the Reynolds number where the skin friction reaches its turbulent level.
\end{abstract}

Masaharu Matsubara

mmatsu@shinshu-u.ac.jp

1 Department of Mechanical Systems Engineering, Shinshu

University, Nagano, Japan

2 Wallenberg Wood Science Center, Department of Engineering Mechanics, KTH Royal Institute of Technology, 10044 Stockholm, Sweden

3 FLOW, Department of Engineering Mechanics, KTH Royal Institute of Technology, 10044 Stockholm, Sweden 


\section{Graphic abstract}

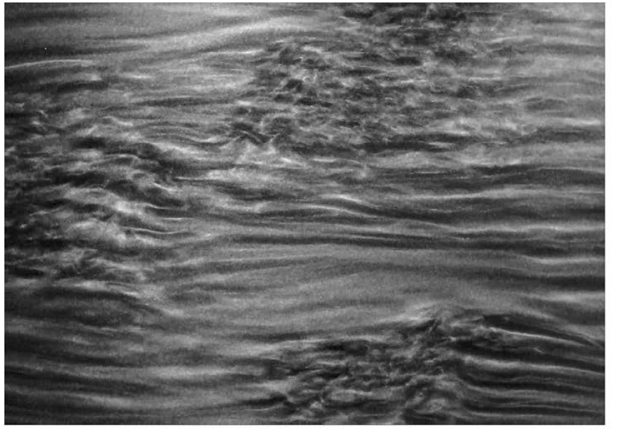

(a)

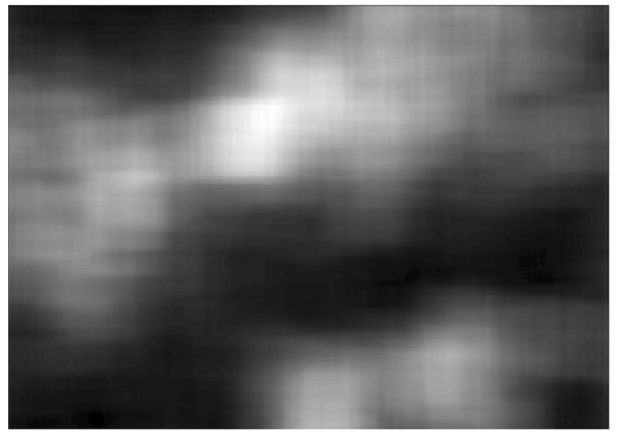

(c)

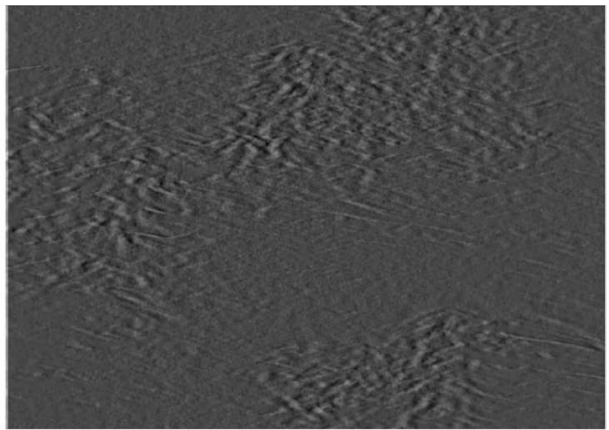

(b)

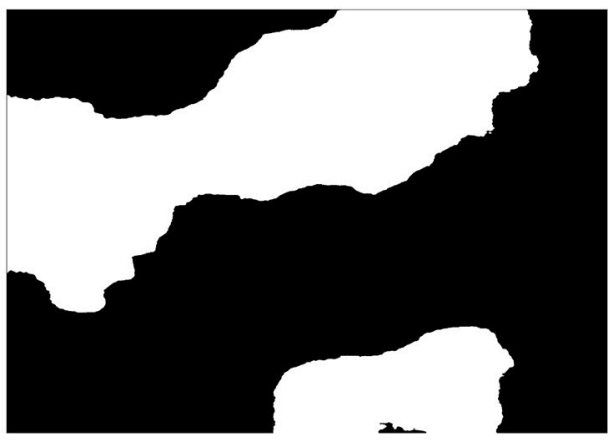

(d)

Processing of flow visualization images to determine the turbulenceintermittency in the transitional region. Flow is from left to right, (a)unprocessed, (b) filtered (c) standard deviation of (b), (d) binary picture

\section{Introduction}

The phenomenon of transition to turbulence in shear flows depends not only on the stability of the flow itself but also on initial, upstream and background disturbances. The stability of the flow can be obtained through linear stability theory where the growth (or decay) of infinitesimal disturbances can be determined. The critical Reynolds number is defined as the lowest Reynolds number for which infinitesimal disturbances amplify. However, it is well known that both Poiseuille pipe and planar Couette flows, for which the critical Reynolds number is infinite, can become turbulent at rather small Reynolds numbers even without introducing external disturbances; the background noise of the facility will be sufficient to trigger transition.

In the last two decades, many researchers made efforts to determine the minimum Reynolds number, sometimes called the transition Reynolds number, for several canonical wall-bounded flows to sustain a turbulent state and to reveal phenomena in moribund turbulence, especially for pipe and plane Couette flows. For instance Avila et al. (2012) estimated the lower $R e$ limit for a sustainable puff in circular pipe flow to 2040 from the crossover of the double exponential functions of puff decaying and separation times obtained experimentally and numerically. In plane Couette flow experiments by Tillmark and Alfredsson (1992) and DNS by Lundbladh and Johansson (1991) the Reynolds number for which turbulence could sustain itself is around 360 (based on half-channel height and half velocity difference between the walls).

In contrast to circular pipe flow and planar Couette flow the critical Reynolds number for planar channel flow is finite with $R e_{\mathrm{CL}, \text { crit }}=5772$ (Orszag 1971), where $R e_{\mathrm{CL}}=U_{\mathrm{CL}} h / \mathrm{v}$ and $U_{\mathrm{CL}}$ is the centreline velocity of the corresponding laminar parabolic profile, $h$ is the channel half height and $v$ the kinematic viscosity of the fluid. Experimentally it is found that planar channel flow usually turns into turbulence below this value and it has been observed that patches filled with turbulence appear intermittently and depending on the Reynolds number they may split, grow or decay as they move downstream (Carlson et al. 1982; Alavyoon et al. 1986; Hashimoto et al. 2009).

Studies on the lowest Reynolds number where turbulent channel flow exists can be traced back to the experiment of Davies and White (1928). They made careful pressure-drop 
measurements in a height-adjustable water-channel facility. The channel had sharp corners at the inlet that generate large amplitude disturbances. The skin friction coefficient was found to have a slight departure from laminar theory above $R e=U_{\mathrm{b}} d / v=890\left(U_{\mathrm{b}}\right.$ is the bulk velocity and $d$ is the channel height, $\left.d=2 h, R e=4 / 3 R e_{\mathrm{CL}}\right),{ }^{1}$ and above $R e=1440$ it gradually increases in contrast to the rapid increase in transitional pipe flow. Badri Narayanan (1968) measured the velocity fluctuations in channel flow and estimated that the minimum Reynolds number at which the turbulent energy is sustained along the streamwise direction of the channel is $R e=2800$. In extensive experiments by Patel and Head (1969), traces of the streamwise velocity fluctuation measured by a hot-wire anemometer indicate that intermittency occurs in the range of $1380 \leq R e \leq 1800$ and it was found from a skin friction coefficient plot that the transitional regime starts around $R e=1350$ and ends somewhere in the range 2500-3000.

Carlson et al. (1982) used a water channel to make flow visualizations of turbulent spots that were generated by a controlled point-like disturbance. They found that below $R e_{\mathrm{CL}}=1000$ the disturbance decays transforming into long streamwise oriented structures. The flow visualisations shown of the generated spots were limited to $R e_{\mathrm{CL}}=1000$ and 1100 and in these cases spot splitting, where the centre of the original spot relaminarizes and gives two spots separated by a region of streaky structures, was observed at some downstream position. A spot-like patch from natural transition at $R e_{\mathrm{CL}}=1200$ was also shown. Another spot visualisation study in a water channel, using a similar triggering technique as that of Carlson et al., but with a wider range of Reynolds numbers $\left(1100<R e_{\mathrm{CL}}<2200\right)$ was made by Alavyoon et al. (1986). In that experiment the minimum $R e_{\mathrm{CL}}$ for which a turbulent spot could be generated was $R e_{\mathrm{CL}}=1100$. They also observe spot splitting at $R e_{\mathrm{CL}}=1100$ though no splitting is found for $R e_{\mathrm{CL}}>1200$. They also determined the propagation speed of the front and rear of the spot as well as the lateral spreading rate as function of Reynolds number, the latter was found to increase linearly with $R e$. Above $R e_{\mathrm{CL}}=2200$ they observed randomly occurring spots in the channel.

In the two previously described flow visualization studies the channel inlet flow was controlled to have a low turbulence level so it was possible to introduce a deterministic disturbance in a laminar flow to generate turbulent spots. Another flow visualization study by Hashimoto et al. (2009) had another approach by instead placing a grid at the channel inlet to generate inlet turbulence. In that case turbulent oblique regions, called stripes by the authors, were observed

\footnotetext{
${ }^{1}$ Here both these two definitions of the Reynolds number (Re and $R e_{\mathrm{CL}}$ ) will be used following the usage in the original paper.
}

in the range 1700-2000, and the observed inclination angle of the stripes was $20^{\circ}-30^{\circ}$ and their streamwise width around $60 \mathrm{~h}$. They found the transition Reynolds number to be 1300 . Sano and Tamai (2016) also performed flow visualization of a transitional channel flow and expressed their results in terms of the intermittency factor $(\gamma$, i.e. turbulent fraction of the flow area, where " 0 " denotes no turbulence and " 1 " denotes fully turbulent). They found that the transitional region started, i.e. $\gamma$ increased from zero, at $R e=1070$.

Nishioka and Asai (1985) performed hot-wire measurements in an air-channel-flow facility where the flow was strongly disturbed by a cylinder (with a diameter somewhat smaller than the channel height) placed between the channel walls or by a jet from an orifice in the channel wall. As indicators of transition they used the appearance of intermittency in the velocity signals and the decrease of the centreline velocity from its laminar value and found that these signs cannot be observed for $R e_{\mathrm{CL}}<1000$, indicating that turbulence cannot be sustained below this value.

A more recent hot-wire experiment was made by Seki and Matsubara (2012) which shows that energy of the streamwise velocity fluctuations decays below $R e=1300$ and ceases to decay above $R e=1400$. A so called 'single slope method' was developed to obtain the intermittency factor without determination of a threshold and they presented a curve of the intermittency factor as function of Reynolds number. They found that the upper marginal $R e$ defined as the lowest $R e$ for fully developed turbulent channel flow is 2600 , and that the lower marginal $R e$ defined as the lowest $R e$ for intermittent channel flow with laminar and turbulent parts is 1400 (this would correspond to the transition Reynolds number). They also revealed that the low-frequency velocity fluctuations with a length scale corresponding to the size of the turbulent patches exist even at $R e=2660$, i.e. above the Reynolds number for which the flow is expected to be fully turbulent.

Watanabe et al. (2012) and Miyazaki (2014) demonstrated that the intermittency factor evaluated from flow visualization gradually increases from $R e=1400$ and asymptotically approaches unity around $R e=2500$. This gradual increase of the intermittency was also quantitatively confirmed by the hot-wire measurements by Seki and Matsubara (2012).

An early direct numerical simulations (DNS) of transitional channel flow was performed by Orszag and Kells (1980). Though the domain size of the simulations was limited because of the low computing power at the time, they confirmed a sustainable disturbed channel flow at $R e=1250$ and suggested that this could even exist at somewhat lower $R e$, but not as low as $R e=500$.

Tsukahara et al. (2014) found that turbulent regions in their DNS at $R e=2320$ are confined to inclined bands. The inclination of the turbulent band with respect to the streamwise direction is $24^{\circ}$ and corresponds to the ratio 
of the spanwise and streamwise domain sizes. They also observed a sustained transitional flow at $R e=1860$. Another DNS by Tuckerman et al. (2014) used an inclined $\left(24^{\circ}\right)$ computational domain in order to specifically simulate the inclined bands. Their figure 3 shows bands in the range $1060<R e<2800$ where for the higher Reynolds numbers the banded structures are coexisting with turbulence that fills the whole domain. ${ }^{2}$ The highest $R e$ in their case with a clear demarcations (laminar-like region) between the bands is $R e=2400$ (their $R e=1800$ ), a value that agrees well with the results of Tsukahara et al.

Another DNS study reported by Shimizu and Manneville (2019) with a very large computational domain was carried out for several Reynolds number in the transitional region. That study shows clearly banded structures in the range $1052<\operatorname{Re}<2457$ (see figure 1 in Shimizu and Manneville $(2019)^{3}$ ) but turbulent spots cannot be observed. At $R e \approx 1349$ an 'intermittent loose continues network' of localized turbulent bands (LTBs) appears. Lowering the Reynolds number to 1263 gives rise to propagating, inclined LTBs that appear as a symmetrical bifurcation (two-sided flow).

The transition scenario in these DNS studies is different from the one in most experiments and this may depend on three causes: (1) different levels of inlet disturbances; (2) the channel aspect ratio. Although the spanwise aspect ratio of most experimental channels is quite large, it is still smaller than the aspect ratio of the modern DNS studies mentioned above. Furthermore the periodic boundary conditions applied in the simulations effectively make the aspect ratio infinite; (3) The finite length of experimental channels only allows flow structures to develop for a certain time, whereas the DNS channel can be viewed as having an infinite length due to streamwise periodic boundary conditions.

The purposes of the present study are to experimentally determine the transitional Reynolds number for physically realizable channel flows and to reveal the transitional state using pressure drop measurements to determine the skin friction as well as through flow visualization. The intermittency factor $(\gamma)$ is evaluated from the flow visualization and is quantitatively determined for a range of Reynolds numbers. Section 2 gives the details of the experimental setup, the pressure-drop measurements and flow visualization equipment. The skin friction coefficient is compared with previous results in Sect. 3 and flow-visualization photographs are shown and discussed in Sect. 4. Section 5 describes the methodology for evaluating the intermittency and the results

\footnotetext{
2 Their $R e$ is recalculated to adhere to the present definition of $R e$ by multiplying with $4 / 3$.

${ }^{3}$ We are here converting the bulk Reynolds number, $R e_{\mathrm{b}}$ in their paper as $R e=4 / 3 \operatorname{Re}_{\mathrm{b}}$.
}

are presented and discussed. Finally Sect. 6 summaries and gives the conclusions of the present work.

\section{Experimental setup}

The experiment was conducted in a water-channel facility shown schematically in Fig. 1. A pump circulates the water (temperature for all measurements $20 \pm 1{ }^{\circ} \mathrm{C}$ ) around a closed flow loop and downstream the pump an orifice for pressure-drop measurements to determine the flow rate $(Q)$ is located. This is followed by an upstream settling box with screens to dampen fluctuations, a two-dimensional nozzle with a contraction ratio of $25: 1$, the channel and a downstream box. The channel walls are vertical and the total length of the channel section is $7320 \mathrm{~mm}$.

Transition to turbulence is triggered by a tripping wire of $0.9 \mathrm{~mm}$ diameter and $220 \mathrm{~mm}$ length placed on one wall of the channel, $290 \mathrm{~mm}$ downstream from the channel entrance. The distance between the side walls changes from 580 to $360 \mathrm{~mm}$ giving an increase in the Reynolds number by $60 \%$. In the narrow channel section, the flow develops into a state of turbulence or highly intermittent flow. The side-wall distance returns to $580 \mathrm{~mm}$ after an expansion section. The coordinate system is defined with $x$ in the streamwise direction and $x=0$ is at the end of the expansion section. The spanwise coordinate is $z$ with $z=0$ at the channel centreline.

The channel test section width is $w=580 \mathrm{~mm}$ and the length $4650 \mathrm{~mm}$. The channel walls for the final $4360 \mathrm{~mm}$ are made from $10 \mathrm{~mm}$ thick glass plates. Each wall is supported by four horizontal beams of aluminum and those in turn are connected to eight vertical beams at each side along the channel. The channel height was measured by an ellipse gauge and was carefully adjusted by inserting shim plates between the lateral and horizontal beams. The average channel height $d$ was $7.02 \mathrm{~mm}$ for $2380 \leq x \leq 4420 \mathrm{~mm}$ within $\pm 1.5 \%$.

A static-pressure tube with an outer diameter of $2.1 \mathrm{~mm}$ is used for measurements of the pressure drop. It has three pressure holes each with a diameter of $0.5 \mathrm{~mm}$ positioned $25 \mathrm{~mm}$ downstream from the rounded tube top. The static tube is supported by an aluminum pipe of $7 \mathrm{~mm}$ diameter and $2500 \mathrm{~mm}$ length which is horizontally inserted into the channel from the end of the downstream box and is traversed manually along $x$. The reference pressure for the statistic pressure measurements is taken at $x=4400 \mathrm{~mm}$ from a pressure tap in the upper side wall. The pressure transducer (LPM5480 GE Druck) has a range 0-1000 Pa and an accuracy (combined non-linearity, hysteresis, and non-repeatability) of $\pm 0.25 \%$.

The flow visualization images/movies are taken with the image centre at $x=3000 \mathrm{~mm}(x / d=427)$ and $z / d=0$. Pearl flakes of $3.1 \mathrm{~g} / \mathrm{cm}^{3}$ density and $7 \mu \mathrm{m}$ size were used as flow 

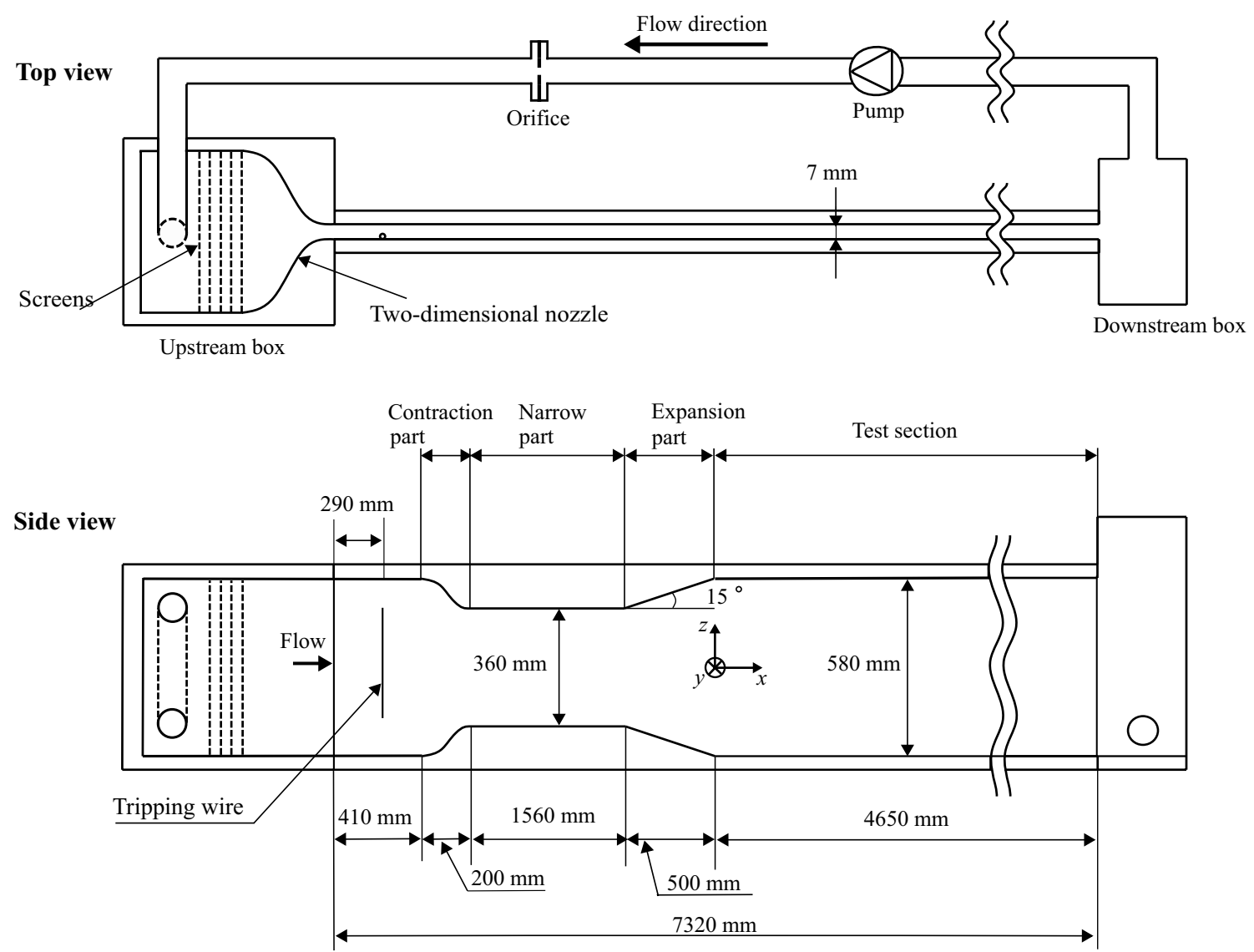

Fig. 1 The experimental water channel transition facility located at the Fluid Dynamics Laboratory of Shinshu University in Nagano. The total length of the flow system is approximately $9 \mathrm{~m}$ and the channel

tracers. Two halogen lamps of $500 \mathrm{~W}$ illuminate the flow at a lighting angle of $50^{\circ}$ measured from the horizontal direction. A high-resolution camera takes pictures $\left(4912 \times 3264 \mathrm{px}^{2}\right)$ and records video sequences $\left(1920 \times 1080 \mathrm{px}^{2}\right)$ of the flow, where a value before ' $x$ ' denotes the streamwise direction and the one after the spanwise direction.

\section{Skin friction coefficient}

The skin friction coefficient $C_{\mathrm{f}}$ is obtained from the measurement of the streamwise pressure gradient $\mathrm{d} P / \mathrm{d} x$ as,

$C_{\mathrm{f}}=\frac{\tau_{\mathrm{w}}}{\frac{1}{2} \rho U_{b}^{2}}=\frac{d}{\rho U_{b}^{2}}\left(-\frac{\mathrm{d} P}{\mathrm{~d} x}\right)$,

where $\tau_{\mathrm{w}}$ is the wall shear stress and $\rho$ the density of water. The bulk velocity $U_{\mathrm{b}}$ is obtained from the measured flow rate $Q$ divided by the channel cross sectional area $A_{\mathrm{ch}}=w d$. It is also convenient to define the friction velocity $u_{\tau}=\sqrt{\tau_{\mathrm{w}} / \rho}$ and the Reynolds number based on friction velocity and half channel height such that $R e_{\tau}=u_{\tau} d / 2 v$. test section is oriented vertically. For more details of the setup and measurement techniques see Sect. 2

In Fig. 2, the variation of the static pressure, divided by $R e$, along the test section is shown for different $R e$. The static pressure distributions give straight lines with a maximum deviation of less than $1.8 \%$ of the pressure difference between $x=3000 \mathrm{~mm}$ and $4200 \mathrm{~mm}$. This high accuracy of the pressure drop is due to the use of a single static pressure tube, in contrast to using pressure taps on the wall. Such taps usually give pressure shifts because of small individual differences of the geometry of the openings of each tap. The straight line for each $R e$ also indicates that the distance between the channel walls is constant and that the flow is fully developed. With the normalisation used $(\Delta P / R e)$ the slope of static pressure distributions should be the same if the flow is fully laminar and as can be seen this is the case for $R e \leq 1280$. The slope departs from the laminar value at $R e=1400$ and the slope at $R e=3790$ has more than doubled as compared to that in the laminar region.

The skin friction coefficient $C_{\mathrm{f}}$ obtained from the pressure drop is shown in Fig. 3. A wide Reynolds number range (more than three orders of magnitude) is shown in Fig. 3a covering the laminar, transitional and fully turbulent regions, whereas Fig. $3 b$ expands the figure around the transitional 
Fig. 2 Streamwise distributions of $P / \operatorname{Re}(\mathrm{Pa})$

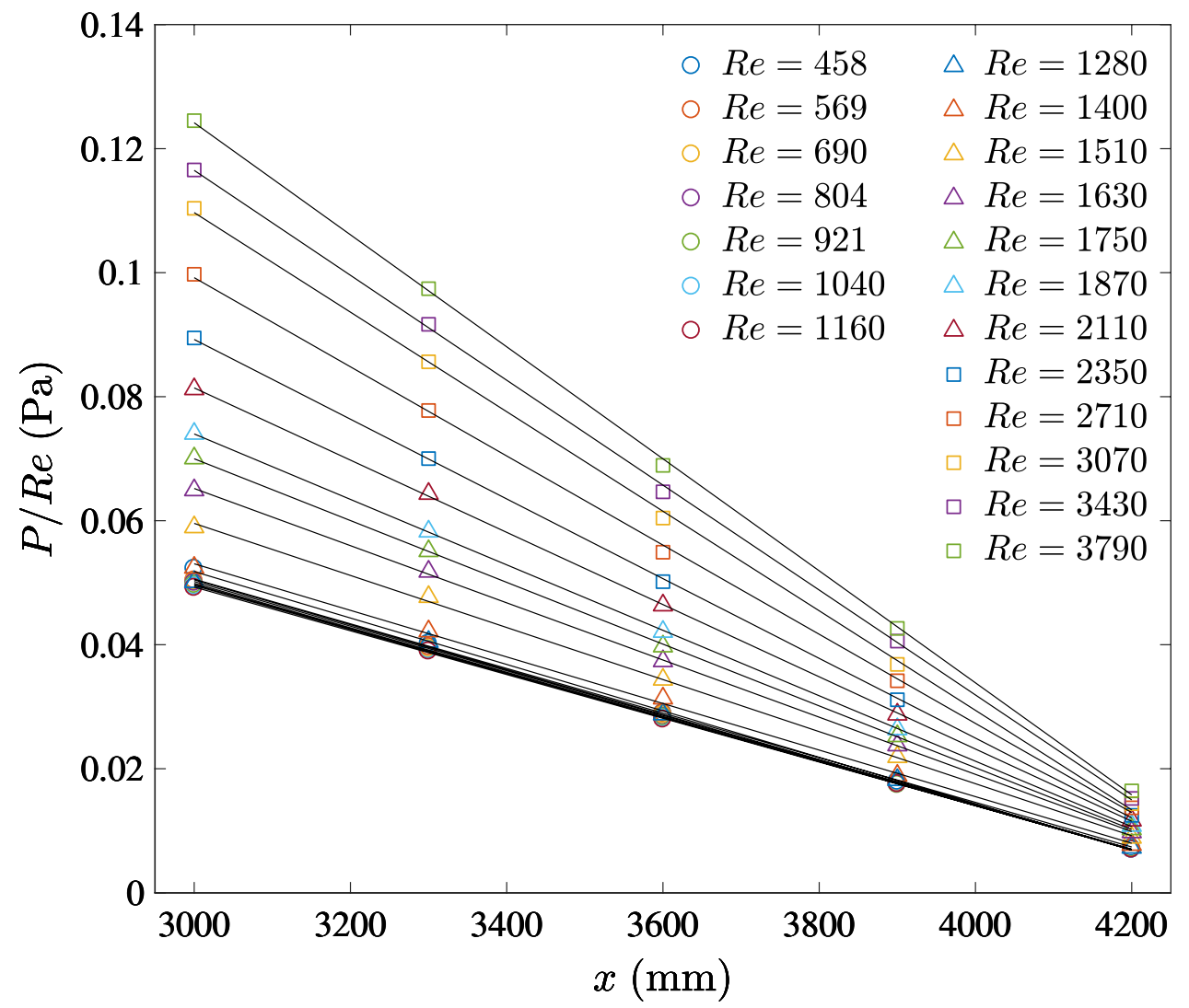

region $(1000<R e<7000)$. For low $R e$ the present data follow the theoretical laminar line for a two-dimensional channel $\left(C_{\mathrm{f}}=12 / R e\right)$. The data starts to deviate from this line around $R e=1250$ and reaches a local maximum around $R e=1900$. Flow visualization results, shown later, demonstrate that the flow is close to fully turbulent for $R e \geq 2600$.

The departure from the laminar $C_{\mathrm{f}}$ line for $R e \gtrsim 1250$, is at a lower $R e$ than in the experiments by Jones (1976) and Patel and Head (1969). However in both those experiments the upstream disturbance was not controlled. In turn, the present data indicate that even if the upstream disturbance is strong the flow still keeps its laminar state for $R e<1250$. As can be seen in Fig. $3 \mathrm{~b}$ the present $C_{\mathrm{f}}$-values above $R e=2000$ are in good agreement with the DNS result by Tsukahara et al. (2014) and the experiment by Jones (1976), although the latter data show some scatter.

The lines in the turbulence region in Fig. 3 are from a Prandtl-Kármán plot shown in Fig. 4. The implicit equation for the friction factor of turbulent pipe flow proposed by Prandtl (1933) is

$\frac{1}{\sqrt{\lambda}}=2.0 \log \left(\operatorname{Re}_{D} \sqrt{\lambda}\right)-0.8$,

where $\lambda$ is the friction factor that equals $4 C_{\mathrm{f}}$ and $R e_{D}=U_{\mathrm{b}} D / v$ is the Reynolds number using the pipe diameter $D$ as the length scale. Jones (1976) found that if this equation is applied to channel flow a laminar equivalent diameter $D_{\mathrm{L}}$ is more appropriate for accurate estimation of $C_{\mathrm{f}}$ as compared to the hydraulic diameter $D_{\mathrm{H}}$. The laminar equivalent diameter is obtained so that the pressure drop of laminar flow in a duct, which can have any cross-sectional shape, has the same pressure drop as a pipe with circular cross section with a diameter $D_{\mathrm{L}}$. For a high aspect-ratio channel $D_{\mathrm{L}}=4 / 3 d$, a value that is smaller than the hydraulic dimeter $D_{\mathrm{H}}=2 d$. Equation 2 with $D=D_{\mathrm{L}}$ reduces to,

$\sqrt{\frac{1}{C_{\mathrm{f}}}}=a \log \left(\operatorname{Re} \sqrt{C_{\mathrm{f}}}\right)+b$,

where the coefficients $a$ and $b$ from several studies are listed in Table 1. The values given by Jones (1976) are obtained from Eq. 2 replacing $D$ by $D_{\mathrm{L}}=4 d / 3$ in the expression for the Reynolds number. Note that $R e$ in Eq. 3 is still defined as $R e=U_{\mathrm{b}} d / v$. Other estimates of the coefficients, based either on a collection of data from several independent experiments (Dean 1978) or from a specific facility (Monty 2004; Zanoun et al. 2009; Schultz and Flack 2013) are also listed. Note that in all these cases the fitting was done for experimental data that span a large $R e$ range. Also the data from Durst et al. (1996) are included in Figs. 3 and 4. 
Fig. 3 Reynolds number dependence of skin friction coefficient $C_{\mathrm{f}}$. a Showing data over a large Reynolds number range; $\mathbf{b}$ same data as in $\mathbf{a}$ focussing on the transitional Reynolds number range. The lines are from Eq. 3 with constants from Table 1 . The chaindotted line is obtained from Eq. 5 and discussed in Sect. 5
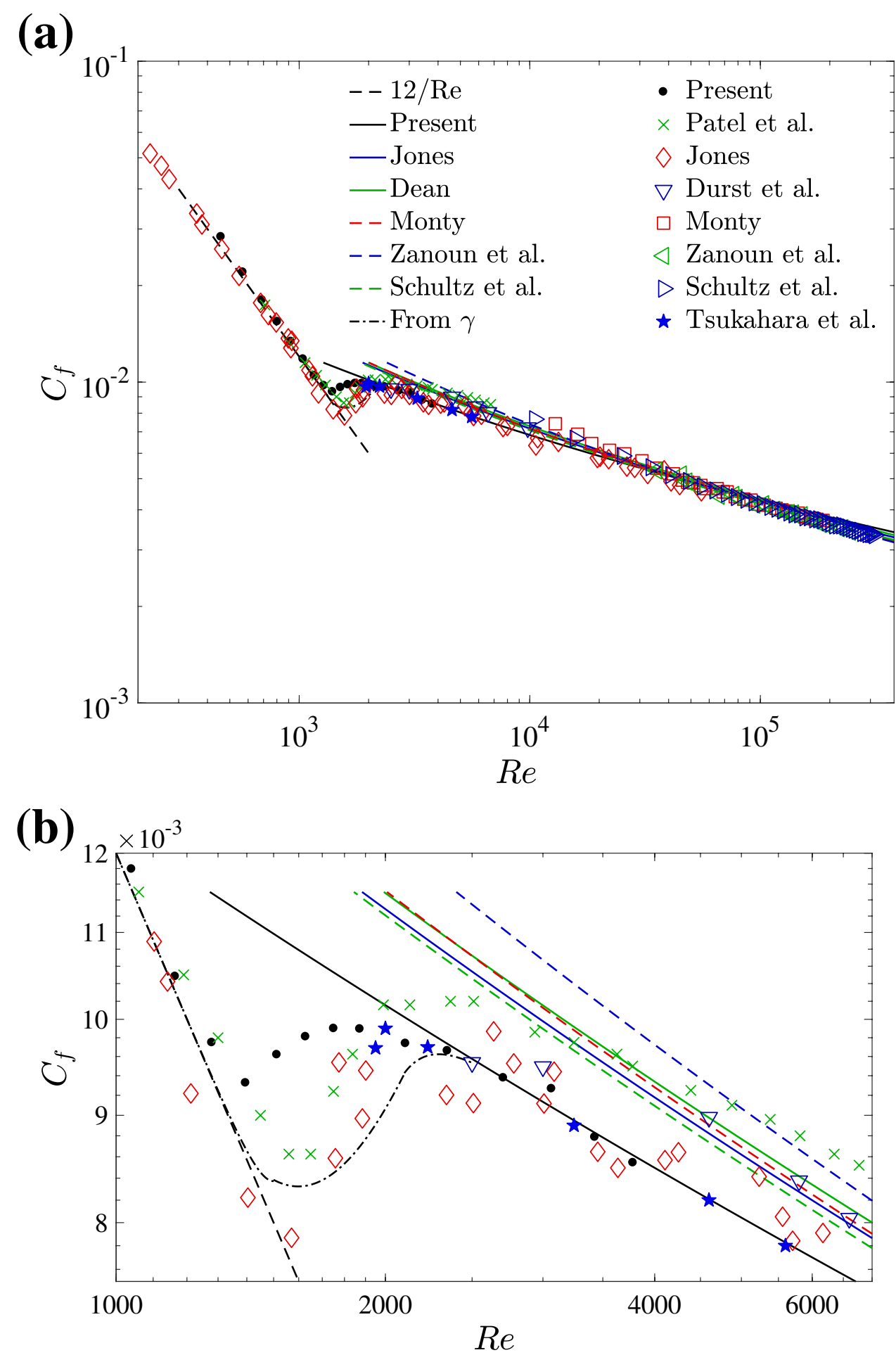

In the Prandtl-Kármán plot shown in Fig. 4, one finds that for all five different choices of the previously defined coefficients from Table 1 the correlations based on Eq. 3 collapse well at high $\operatorname{Re}$ (Fig. 4a), where the present set of coefficients shows the largest difference. This is no surprise since the present coefficients are obtained for data within a narrow range of low Reynolds numbers $(2350<R e<3800)$.
It may be interesting to note that the slope $a$ is directly connected to the Kármán constant $\kappa$. The average value of the inclination $a$ determined from the five wide-range $R e$ data sets in Table 1 is 4.1 , giving $\kappa=\ln 10 /(\sqrt{2} a)=0.40$.

Figure $4 \mathrm{~b}$ shows that the present data for $\sqrt{1 / C_{\mathrm{f}}}$ are in agreement with the DNS by Tsukahara et al. (2014) and the experimental results of Jones (1976), for $\operatorname{Re} \sqrt{C_{\mathrm{f}}}>200$ 
Fig. 4 Prandtl-Kármán plot, same data as in Fig. 3. a Showing data over a large Reynolds number range; $\mathbf{b}$ same data as in a focussing on the transitional Reynolds number range. The lines are from Eq. 3 with constants from Table 1
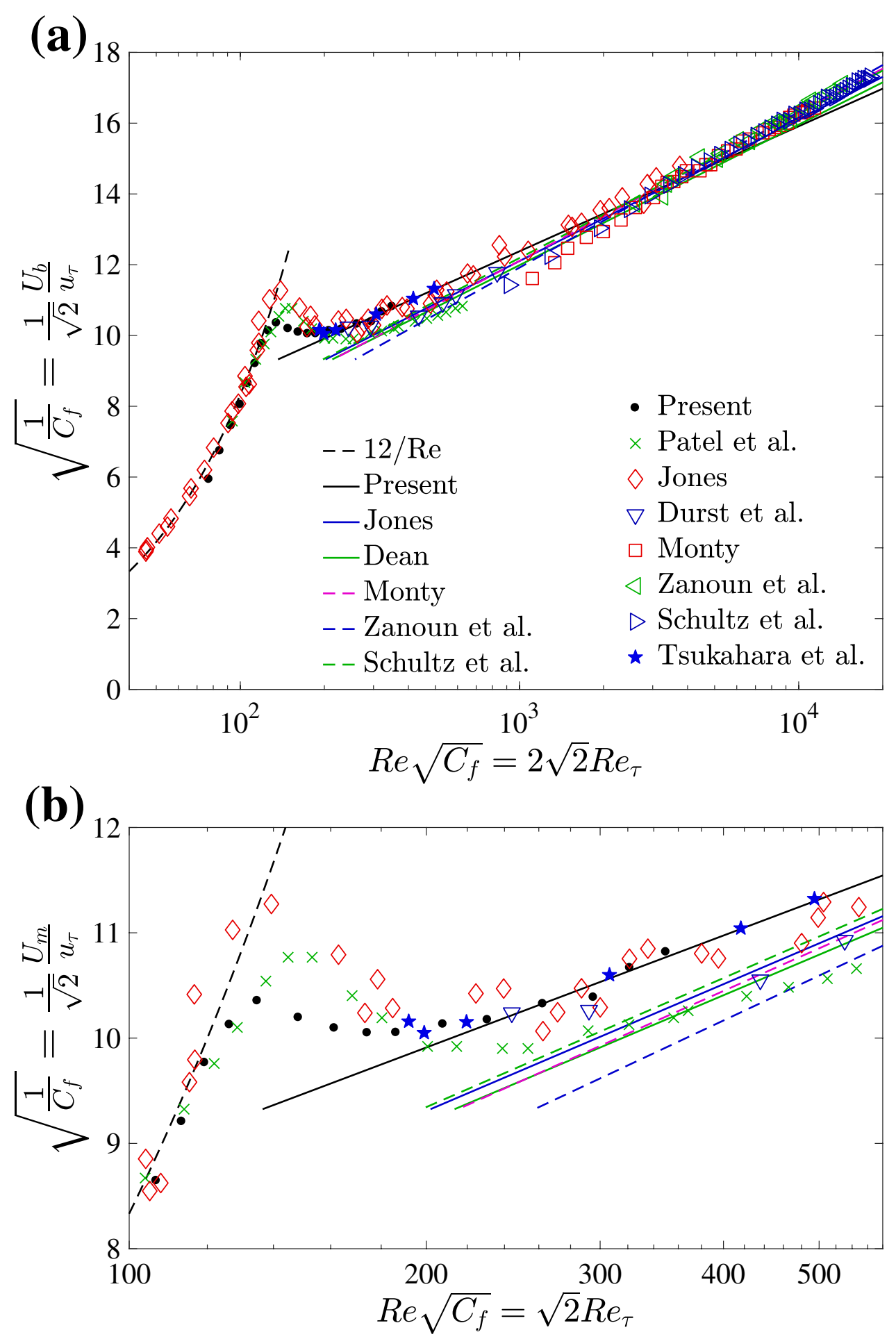

(approximately corresponding to $R e=2000$ ), as also can be expected from the results shown in Fig. 3b. They are all shifted up compared to the five lines (see Fig. 4b) and a corresponding downward shift (approximately 10\%) is seen for $C_{\mathrm{f}}$ in the range $2000<R e<4000$ in Fig. 3b. The coefficients for the line adhering to the present data in Fig. 4 are given in Table 1 clearly showing that $C_{\mathrm{f}}$ in the 
Table 1 Coefficients of Eq. (3)

\begin{tabular}{lll}
\hline & $a$ & $b$ \\
\hline Jones (1976) & 4.00 & 0.10 \\
Dean (1978) & 3.97 & 0.074 \\
Monty (2004) & 4.175 & -0.416 \\
Zanoun et al. (2009) & 4.40 & -1.28 \\
Schultz and Flack (2013) & 4.07 & -0.021 \\
Present & 3.53 & 1.78 \\
\hline
\end{tabular}

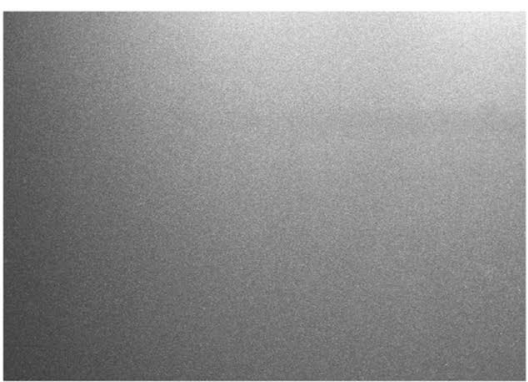

(a)

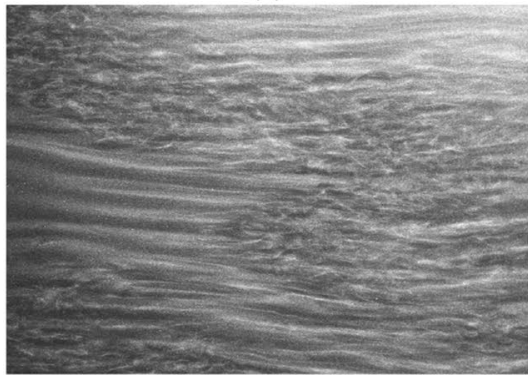

(d)

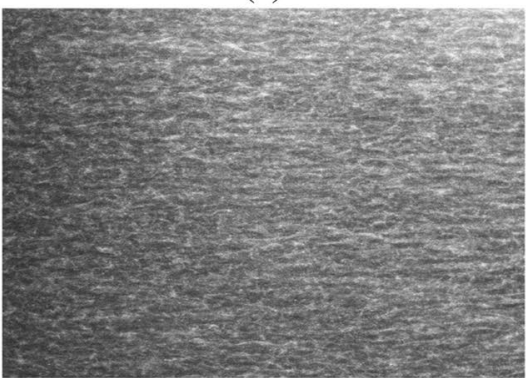

(g)

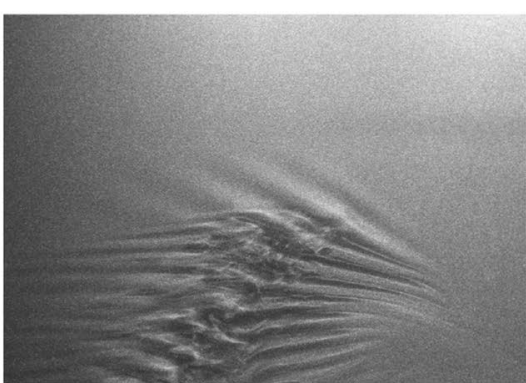

(b)

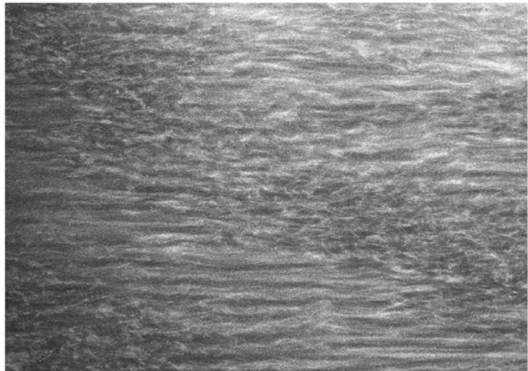

(e)

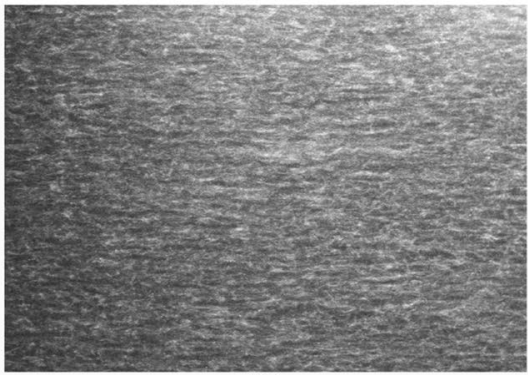

(h) range $1200 \leq R e \leq 3600$. The flow direction is from left to right and the area of the photographs is $184 \mathrm{~mm} \times 123 \mathrm{~mm}$ $(26.2 d \times 17.5 d)$ in the streamwise and spanwise directions, respectively.

Figure $5 \mathrm{a}$ at $R e=1200$ shows a homogeneous image indicating that most tracer particles are oriented in the same way which is a sign that the flow is laminar and that no flow structures are present. Figure $5 \mathrm{~b}$ at $R e=1250$ shows a characteristic turbulent spot in an otherwise laminar flow, though such appearances of spots are rare for this Re. As

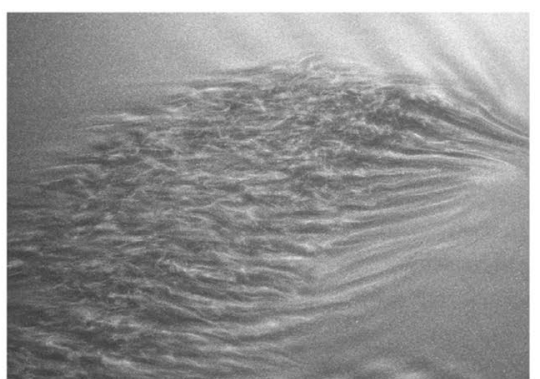

(c)

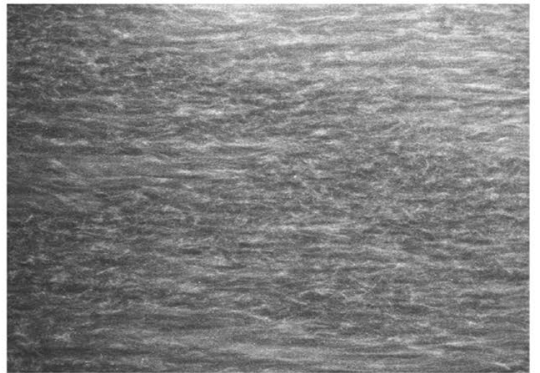

(f)

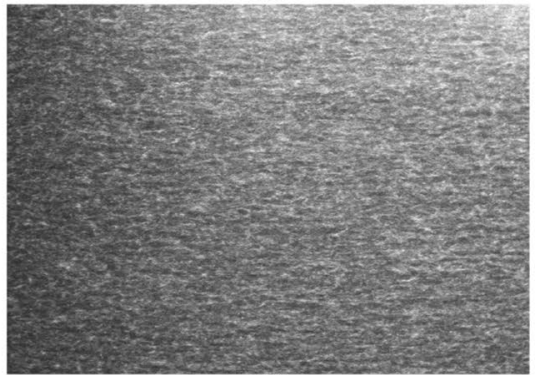

(i)

Fig. 5 Flow visualization in the range $1200 \leq R e \leq 3600$. a $R e=1200 ;$ b $1250 ;$ c $1400 ;$ d $1500 ;$ e 1800; f 2000; g 2600; h 2700; i 3600

low $R e$, but turbulent, region does not obey Eq. 3 with coefficients determined from high Reynolds number data.

\section{Flow visualization}

To determine the Reynolds number range of the transitional region and to survey its characteristics, flow visualizations have been made through photography and video imaging. Figure 5 shows nine flow visualization snapshots in the shown in Fig. 6, this spot shows similar properties to the turbulent spots that Carlson et al. (1982) and Alavyoon et al. (1986) artificially generated by a point-wise disturbance in a laminar channel flow, although in their case it was symmetric with respect to $z=0$. Typical features are an arrowhead formed region containing streaky structures, a tongue of breakdown filled with small-scale eddies, oblique waves beside the tongue and streamwise streaky waves trailing at the rear of the turbulent area. At this Reynolds number 
Fig. 6 Turbulent patch details based on the turbulent spot nomenclature introduced by Carlson et al. (1982). The figure shows the same spot as in Fig. 5b. (1) Patch front (arrow head shape); (2) leading edge; (3) oblique wave; (4) streak wave; (5) region of small-scale turbulence; (6) tongues of breakdown

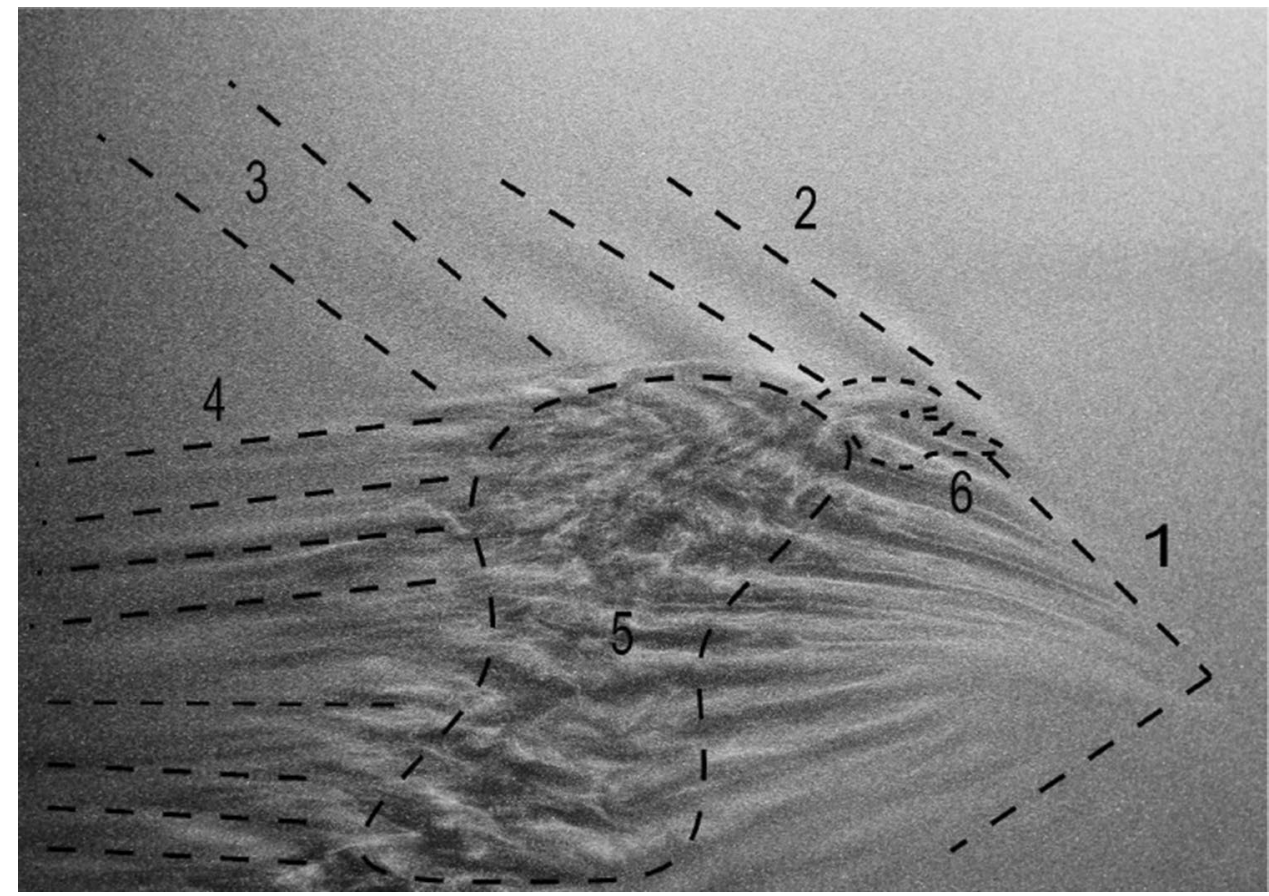

$(R e=1250)$ the $C_{\mathrm{f}}$ data shown in Fig. 3 starts to depart from the laminar line.

At $R e=1400$ in Fig. 5c, the turbulent area with small scale structures increases in size, though the arrowhead shape and the oblique waves are still observed. The flow at $R e=1500$ (Fig. 5d) does however not include oblique waves or a spot-like turbulent area. Instead the flow consists of patchwork-like turbulent areas and areas filled with largescale streaky structures in between. The turbulent patches tend to form oblique bands, clearly observed in the DNS by Tsukahara et al. (2014), though the band patterns here are not that clear. In the DNS the orderly band formation may be enhanced by the streamwise and spanwise periodic boundary condition. Typical streamwise and spanwise sizes of the turbulent patches are about $10 d$ and $5 d$, respectively. The streaky structures around the patches do not include small-scale turbulence.

Long-time observations of the flow visualization show that the turbulent patches deform in time and new such patches do not appear inside the non-turbulent regions, neither do non-turbulent regions appear inside the turbulent patches. This indicates that turbulence generation and extinction only occur around the boundaries of the turbulent and non-turbulent regions.

The series of images in Fig. 5d-h show that the intermittency factor, or turbulent fraction of flow, increases as $R e$ increases. It is hard to distinguish the streaky part in the flow visualization at $R e=2600$, a $R e$ that is close to the upper marginal value defined by Seki and Matsubara (2012) for where the intermittency becomes close to one. The typical streamwise and spanwise scales were determined by eye from enlarged pictures where regions of connected white pixels were used to determine their size. At $R e=2600$ the streamwise and spanwise scales were approximately $1 d$ and $0.5 d$, respectively. At $R e=3600$ (Fig. 5i), the turbulent eddies are homogeneously distributed across the full channel. They also become smaller than those at $R e=2600$ and their streamwise and spanwise sizes are $0.8 d$ and $0.3 d$, respectively.

Careful observation of video visualizations by eye confirmed clusters with dense streaks for $2600 \leq R e \leq 2700$. These are not ordered like streaks around the turbulent patches seen for lower $R e$ in the transitional region. The region marked by the oval in Fig. 7 consists of wavy streaks with a longer streamwise length and slightly intensified brightness compared to those outside this region. The average length of the streaks in the clusters is $7 d$ which is about ten times longer than that of the vortical structure observed in the fully turbulent region. The typical size of such clusters is about $10 d$ in the streamwise direction and $5 d$ in the spanwise direction. These dimensions are almost the same as the patches that appear in the transitional channel flow and the streamwise size is consistent with that of the verylarge-scale structure (VLSS) found from the velocity power spectra of the turbulent channel flow for $2650 \leq R e \leq 4000$ (Matsubara et al. 2016).

Two-point velocity measurements (Tanada and Matsubara 2019) show that VLSS with a spanwise length scale of 6-8d transforms into narrow VLSS with a spanwise size of about $1.4 d$ above $R e=3400$. The size of the VLSS for $R e<3400$ is similar to those of the turbulent patch and corresponds to the spectral peak frequency observed by Matsubara et al. 
Fig. 7 Enlarged flow structures at Reynolds number 2700 in Fig. 5h. Actual size is $14.4 d \times 10.1 d$. The structures inside the red ellipse circle are a band of streaky structures. The blue dashed rectangle is for comparison a region containing incoherent small-scale turbulence

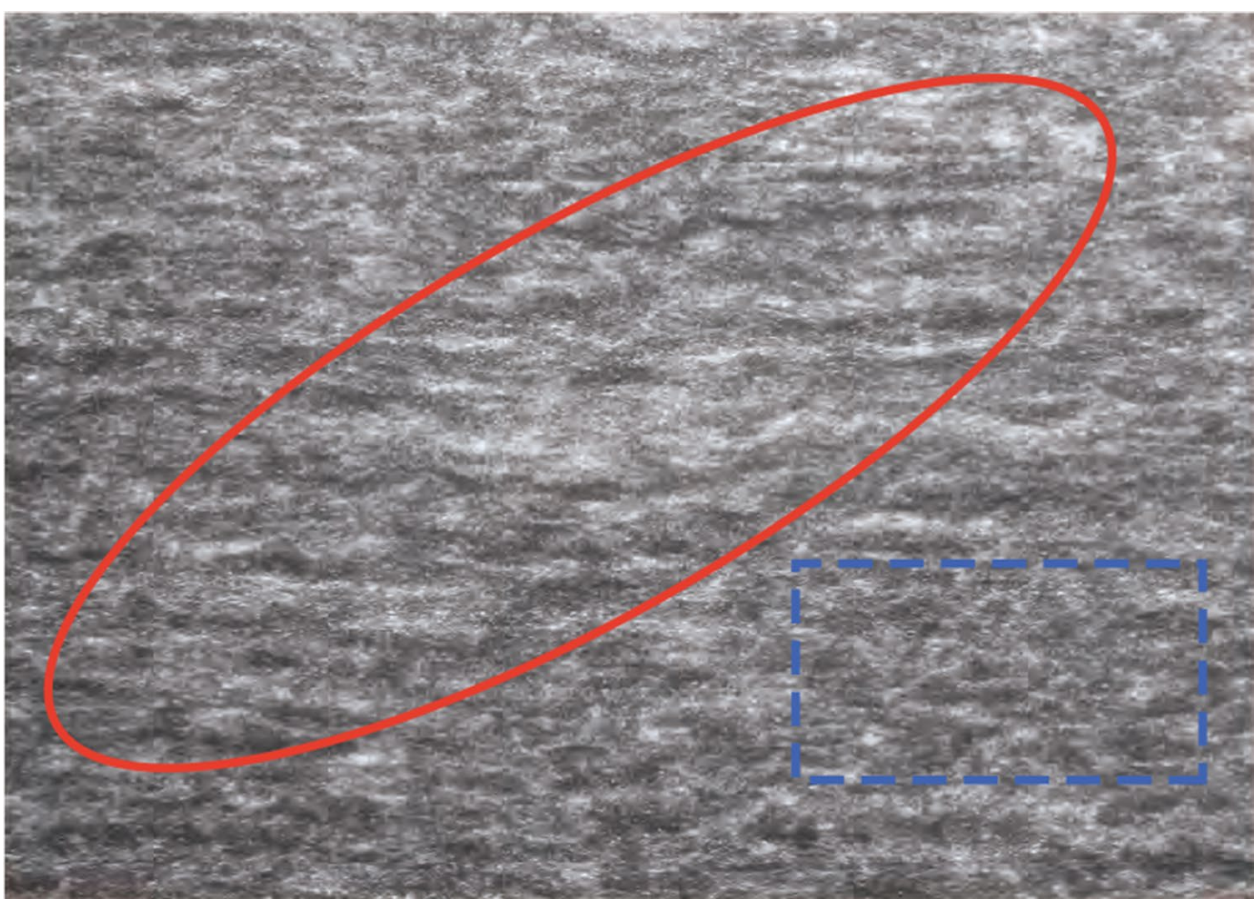

(2016). From these results it is inferred that the non-turbulent regions filled with the streaks observed in the transitional flow still exist even in the fully chaotic flow above $R e=2600$. Therefore, one may consider that transition continues up to $R e=3400$ in the sense that the patch-like flow still exists.

\section{Intermittency factor}

Figure 5 shows that the transitional flow consists of turbulent and non-turbulent parts, so that the intermittency factor $\gamma$, or turbulent fraction, is an appropriate measure of the stage of the transition progress. From the flow visualizations the intermittency factor $\gamma$ can be calculated as the ratio of turbulent areas $A_{\text {turb }}$ to the total picture area, $\gamma=A_{\text {turb }} / A_{\text {total }}$. For the determination of $A_{\text {turb }}$ we utilized an image processing technique detailed below.

An example of an image obtained from a flow visualization movie in the transitional range is shown in Fig. 8a. The flow direction is from left to right and the actual size of the image is $162 \times 92 \mathrm{~mm}^{2}\left(1920 \times 1080 \mathrm{px}^{2}\right)$.

The first step of the process, is to subtract an average of 200 images from each image in order to remove reflections on the glass surface. In the second and third steps, moving averages with two different windows $50 \times 5 \mathrm{px}^{2}$ and $40 \times 5 \mathrm{px}^{2}$ are applied individually and the difference is obtained by subtraction between the moving-average images. The two images where the moving averages have been employed are shown in Fig. 8b,c and by eye almost no differences can be seen between them. However after subtracting them from each other one obtains the image in Fig. 8d with areas of high intensity small-scale fluctuations and other areas with low intensity fluctuations, showing a successful removal of the large-scale streaky structures. The high-intensity areas are interpreted as regions of turbulence. In Fig. 8e, an image of the standard deviation calculated from this subtracted image with a moving widow with $199 \times 199 \mathrm{px}^{2}$ has high values (light areas in the figure) in the turbulent areas, and its binary image shown in Fig. $8 \mathrm{f}$ clearly distinguishes the turbulent areas from the laminar ones.

The threshold for the binarization was first adjusted so that the detection fits to the turbulent areas judged by eye and was given a value of $\theta=0.055$ (this value does not have any physical meaning, but is just a measure related to the evaluation process of the images) and was fixed to the same value for the whole $R e$ range of the present experiments.

Although this procedure may be seen as a subjective determination of the threshold (as pointed out by the reviewers of this paper) a further analysis of the influence of the threshold was carried out, see Fig. 9. The figure includes curves for 19 Reynolds numbers ranging from 1240 to 2860 . By studying the skin friction coefficient in Fig 3b, we would expect that the intermittency for the four lowest Reynolds numbers, 1240 to 1380 , to be close to 0 , which means that $\theta$ should be larger than about 0.058 . With the same reasoning we would expect the four highest $R e, 2480$ to 2860 , to be fully turbulent which would require $\theta$ to be less than about 


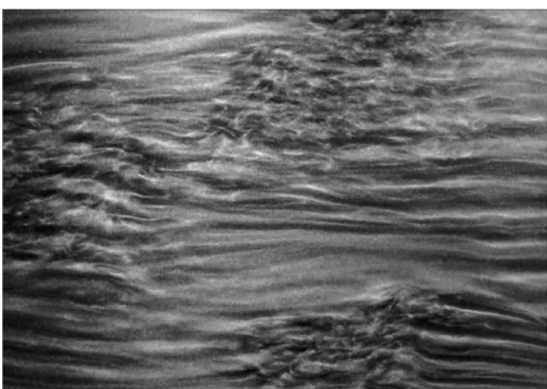

(a)

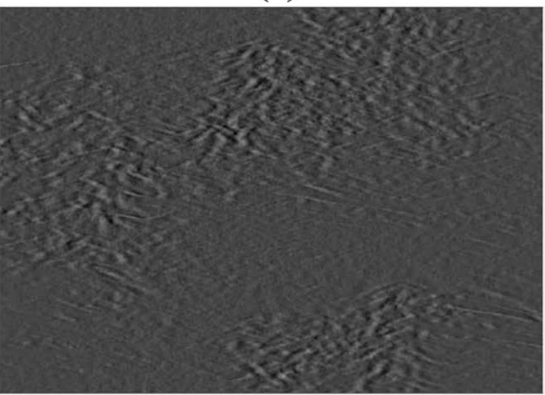

(d)

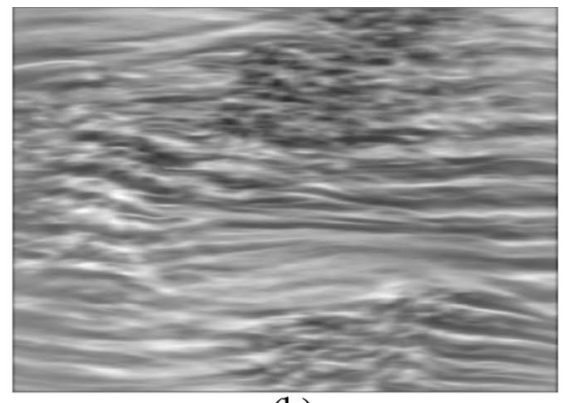

(b)

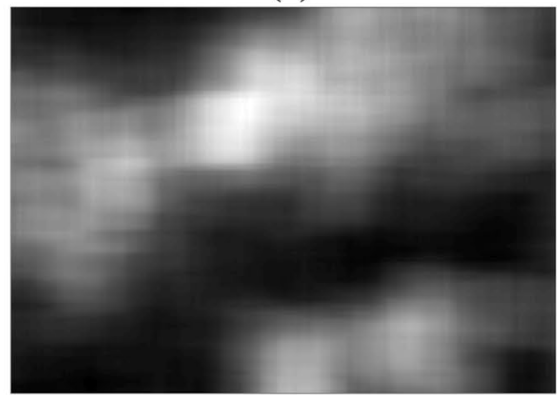

(e)

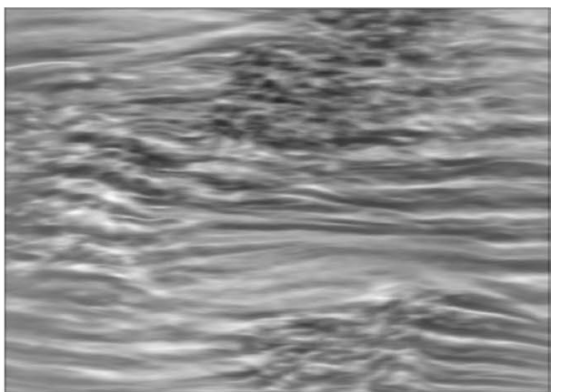

(c)

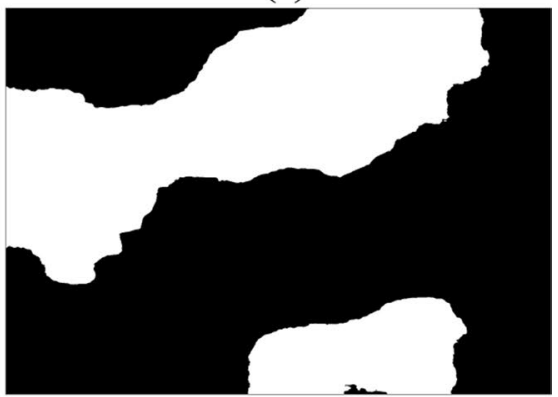

(f)
Fig. 8 Example of image processing used for the estimation of $\gamma$. a original picture; b picture after averaging with window size $50 \times 5 \mathrm{px}^{2} ;$ c picture after averaging with window size $40 \times 5 \mathrm{px}^{2}$; d picture after subtract process of picture (b) and (c); e picture after using standard deviation filter process; $\mathbf{f}$ binary image for laminar or streak (black) area and turbulent area (white)
Fig. 9 The variation of the intermittency factor as function of the threshold level for 19 different Reynolds numbers ranging from 1240 to 2860

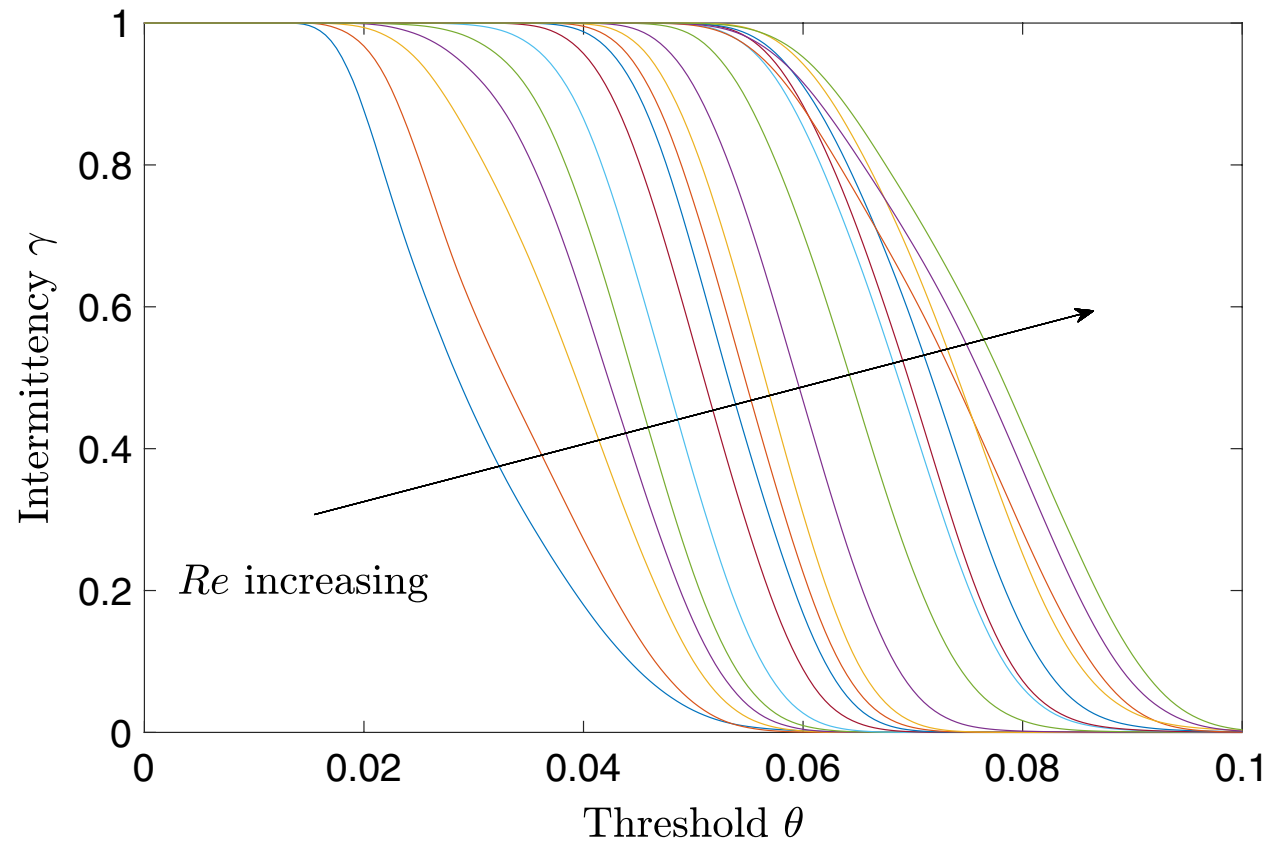

0.052. So with this in mind it seems that original value of 0.055 was a reasonable choice for the threshold.

The intermittency factor $\gamma$, obtained from the image processing, shown in Fig. 10, starts to increase around $R e=1250$ and for $1500<R e<2100$ it increases linearly with $R e$. For $2100<R e<2600$ it asymptotically approaches one. The curve of $\gamma$ is in good agreement with the results by Seki and
Matsubara (2012) who used hot-wire measurements of the streamwise velocity for three different wall distances to detect the turbulent parts of the signal. The fact that the $\gamma$-distributions of the two experiments collapse gives further confidence that the present image processing technique to estimate $\gamma$ is adequate. The experimental data by Sano and Tamai (2016) are also shown in Fig. 10 which show an onset at a lower Re. 
Fig. 10 The intermittency factor $\gamma$ of present data compared with the flow visualization data of Sano and Tamai (2016) and the hot-wire measurements by Seki and Matsubara (2012). The latter is from three different $y$-positions $(y / d=0.3$, 0.4 and 0.5 ), where $y=0$ is at one wall and $y / d=0.5$ is consequently at the channel centreline. The straight blue line is fitted to the data in the range $1500<R e<2100$ and corresponds to Eq. 4. The red line for $R e<1500$ and the green line for $R e>1900$ are fitted lines to the data according to Fig. 11. The dashed line is calculated from Eq. 5 and the measured skin friction coefficient

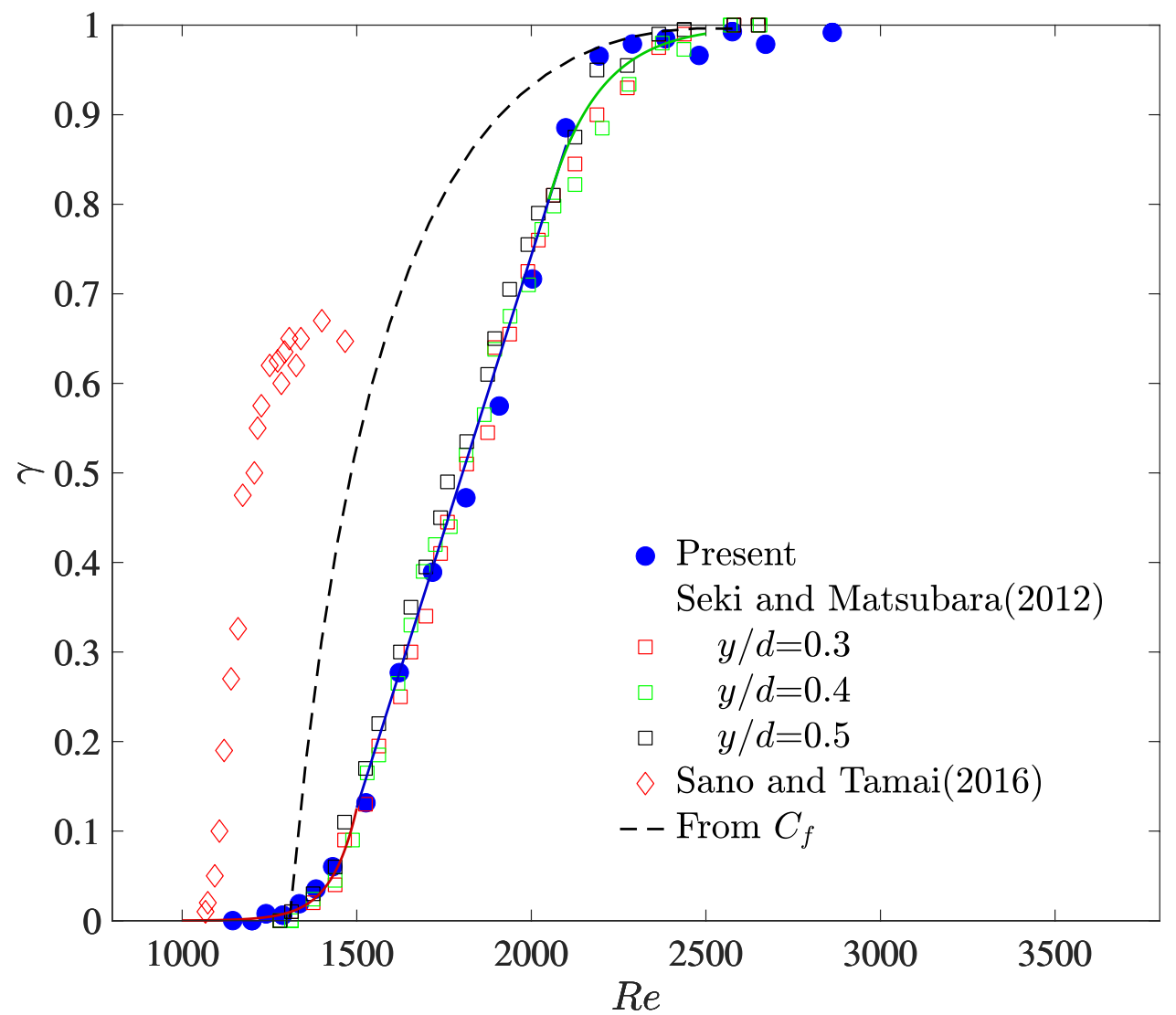

Their results were also obtained by a flow visualization technique but with a detection algorithm using a simple binarization with a low threshold which may have detected fluctuations that may not be related to small-scale turbulence.

For the data in Fig. 10 a straight line can be fitted to $\gamma$ in the region $1500<R e<2100$ giving,

$\gamma=0.001233(\operatorname{Re}-1398)$.

The skin friction coefficient $C_{\mathrm{f}}$ can be estimated from this equation with a simple assumption that local $C_{\mathrm{f}}$ values in the non-turbulent and turbulent regions follow the laminar theoretical and turbulent empirical formulas, $C_{\mathrm{f}, \mathrm{lam}}$ and $C_{\mathrm{f}, \text { turb }}$, respectively, as

$C_{\mathrm{f}}=(1-\gamma) C_{\mathrm{f}, \mathrm{lam}}+\gamma C_{\mathrm{f}, \mathrm{turb}}$.

The chain-dotted line in Fig. 3 is from this estimation with the present coefficients from Table 1 for the turbulent empirical formula and it is seen that the estimated $C_{\mathrm{f}}$ deviates from the laminar line later than the skin friction measurements. This may be explained by the fact that the non-turbulent region is not a pure laminar flow. The streaks in those regions may induce momentum transfer in the wallnormal direction, resulting in an increase of $C_{\mathrm{f}}$. Using the measured skin friction data, i.e. the measured $C_{\mathrm{f}}, \gamma$ can also be estimated by Eq. 5, and is shown in Fig. 10. As expected the modelled intermittency reaches higher levels at low $R e$ as compared to the direct measurements of $\gamma$.

In Fig. 11, $\gamma$ and $\gamma-1$ are plotted in logarithmic scale. An asymptotical approach to zero around the lower marginal $R e$ is seen in Fig. 11a. Though determination of the function of the curve is difficult because of scatter of $\gamma$, it is clear that $\gamma$ decreases in an exponential sense. Around the upper marginal $R e, \gamma$ also approaches one in an exponential sense as seen in Fig. 11b. This asymptotic nature suggests that the probabilistic method represented by the direct percolation (Sano and Tamai 2016; Chantry et al. 2017) may explain and predict the $\gamma$ curves. Though these asymptotes make determination of the marginal Reynolds numbers difficult, a simple estimate by extrapolating the line of Eq. 4 indicates that the lower and upper marginal Reynolds numbers are close to 1398 and 2208, respectively.

For the lower marginal Reynolds number, $R e=1398$, the channel width in wall units is $d^{+}=92$ $\left(d^{+}=u_{\tau \text {,lam }} d / v=\sqrt{6 R e}\right.$, where $u_{\tau \text {,lam }}$ is the friction velocity for laminar flow at this Reynolds number). A streamwise vortex with a diameter of half the channel height then has a size of 46 in wall units, a value that is close to the minimum streak-width Reynolds number of 50 proposed by Alfredsson and Matsubara (2000). This supports the hypothesis that the size of a sustainable vortex cannot be below about 50 
Fig. 11 Asymptotic approach of $\gamma$ around the a lower transitional marginal $R e ; \mathbf{b}$ upper transitional marginal $R e$. The straight red line in a is fitted to the present data and those of Seki and Matsubara (2012) up to $R e=1500$. The green line in $\mathbf{b}$ is fitted to the data of Seki and Matsubara starting at $R e=1900$. The equations for these lines are; red line: $\ln \gamma=0.0135 R e-22.3$, green line:

$\ln (1-\gamma)=-0.00672 R e+12.1$
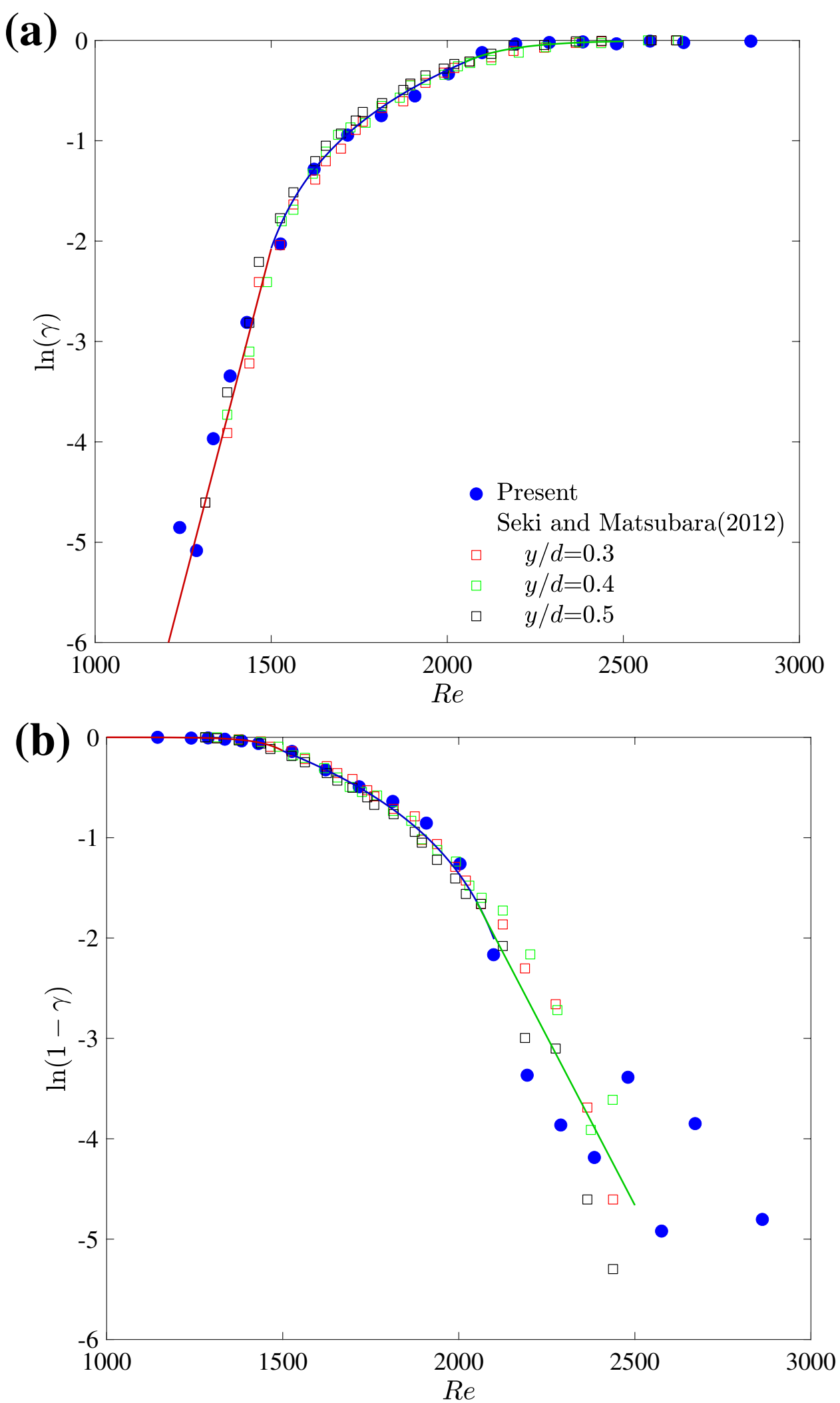

in wall units which is also the typical spanwise size of the streaks observed close to the wall in wall-bounded turbulent shear flows.
The linear increase of $\gamma$ between the asymptotes suggests that there is a balance between turbulent erosion at the boundaries of the turbulent parts and extinction of 
turbulence in other regions. The linear increase of $\gamma$ starts at $R e \approx 1500$ and this is the same Reynolds number at which the flow pass into a patchwork-like state. In this state nonturbulent parts are surrounded by turbulent parts, so that the non-turbulent parts would be continuously subjected to the turbulent erosion around their frontiers.

As seen in the flow visualization at $R e=2700$ in Fig. 7, the streaky regions do not disappear at high Reynolds numbers: the streaks in the clusters seem to be chaotic and cannot be easily distinguished from the turbulent parts that comprise random small-scale structures. The recent hot-wire measurements by Matsubara et al. (2016) demonstrate that a power-spectrum peak of the streamwise velocity fluctuations is observed at $R e=3000$. The frequency of the peak corresponds to a streamwise scale of $25 d$, a scale that is close to twice the streamwise length of the clusters of wavy streaks shown in Fig. 7. If the end of the transition is defined by disappearance of the chaotic streaky regions, the upper marginal $R e$ is higher than that determined by the intermittency factor $\gamma$.

\section{Conclusions}

The determination of the skin friction coefficient and the flow visualization were carried out in order to investigate the transition from laminar to turbulent flow in a wide aspect ratio channel with a strong inlet disturbance. The fact that the transition to the turbulent value of the skin friction coefficient is faster than in previous experiments indicates the importance of a high upstream disturbance level in order to determine the low-end marginal Reynolds number. At a Reynolds number of 1000 turbulent patches were rarely observed in the present flow visualization. This is in accordance with previous experiments (Carlson et al. 1982; Alavyoon et al. 1986) where deterministic jet-injection disturbances were found to be unable to generate sustained turbulent spots below a Reynolds number of 1100.

In the Reynolds number range just above transition, the skin friction coefficient is in good agreement with the experiment by Jones (1976) and the DNS by Tsukahara et al. (2014). However $C_{\mathrm{f}}$ is about $10 \%$ lower than the implicit relations suggested by Jones (1976), Dean (1978), Monty (2004), Zanoun et al. (2009) and Schultz and Flack (2013) based on high Reynolds number data. An attempt to model the skin friction in the transitional region based on the intermittency and the laminar and turbulent skin friction values gives too low values compared to the experimental ones, indicating that the laminar flow regions close to the turbulent patches have a higher skin friction than that of the "clean" laminar flow.

The intermittency factor increases linearly in the Reynolds number range between 1500 and 2100. This suggests that the generation and extinction of turbulence at boundaries between turbulent and laminar regions are in equilibrium, and that the level of intermittency is determined by viscous suppression. On the other hand, the approaches of the intermittency factor to 0 and 1 are both close to exponential. This implies that flows around the marginal Reynolds numbers have a stochastic behaviour.

Though the intermittency factor from the image processing of the flow visualizations shows that the flow becomes fully turbulent at a Reynolds number of 2700, there still exist patch-like areas with dense wavy streaks. The streamwise scale of this streaky patch corresponds to the low-frequency peak observed in the power spectrum of the streamwise velocity measured by Matsubara et al. (2016). This strongly suggests that the Reynolds number at which the transition process is complete, i.e. where the streak clusters have disappeared completely, is higher than those at which the intermittency (turbulent fraction) is one.

Acknowledgements We would like to thank Takayuki Numano for building the experimental setup, and Kenta Watanabe and Makoto Miyazaki for improvements of the techniques for flow visualization and skin friction measurements. Henrik Alfredsson wants to thank Shinshu University for hosting him during the writing of this paper.

Open Access This article is licensed under a Creative Commons Attribution 4.0 International License, which permits use, sharing, adaptation, distribution and reproduction in any medium or format, as long as you give appropriate credit to the original author(s) and the source, provide a link to the Creative Commons licence, and indicate if changes were made. The images or other third party material in this article are included in the article's Creative Commons licence, unless indicated otherwise in a credit line to the material. If material is not included in the article's Creative Commons licence and your intended use is not permitted by statutory regulation or exceeds the permitted use, you will need to obtain permission directly from the copyright holder. To view a copy of this licence, visit http://creativecommons.org/licenses/by/4.0/.

\section{References}

Alavyoon F, Henningson DS, Alfredsson PH (1986) Turbulent spots in plane Poiseuille flow-flow visualization. Phys Fluids 29:1328-1331

Alfredsson PH, Matsubara M (2000) Free-stream turbulence, streaky structures and transition in boundary layer flows. Am Inst Aeronaut Astronaut 2000:2534

Avila K, Moxey D, Lozar A, Avila M, Barkley D, Hof B (2012) The onset of turbulence in pipe flow. Science 333:192-196

Badri Narayanan MA (1968) An experiment study of reverse transition in two-dimensional channel flow. J Fluid Mech 31:609-623

Carlson DR, Widnall SE, Peeters MF (1982) A flow visualization study of transition in plane Poiseuille flow. J Fluid Mech 121:485-505

Chantry M, Tuckerman L, Barkley D (2017) Universal continuous transition to turbulence in a planar shear flow. J Fluid Mech 824:R1

Davies SJ, White CM (1928) An experimental study of the flow of water in pipes of rectangular section. Proc R Soc Lond A 119:92-107 
Dean RB (1978) Reynolds number dependence of skin friction and other bulk flow variables in two-dimensional rectangular duct flow. J Fluids Eng 100:215-222

Durst F, Kikura H, Lekakis I, Jovanovic J, Ye Q (1996) Wall shear stress determination from near-wall mean velocity data in turbulent pipe and channel flows. Exp Fluids 20:417-428

Hashimoto S, Hasobe A, Tsukahara T, Kawaguchi Y, Kawamura H (2009) An experimental study on turbulent-stripe structure in transitional channel flow. In: Hanjalić K, Nagano Y, Jakirlić S (eds) Turbulence, heat and mass transfer, vol 6. Begell House Inc, pp 10

Jones OC Jr (1976) An improvement in the calculation of turbulent friction in rectangular ducts. J Fluids Eng 98(2):173-178

Lundbladh A, Johansson AV (1991) Direct simulation of turbulent spots in plane Couette flow. J Fluid Mech 229:499-516

Matsubara M, Horii S, Sagawa Y, Takahashi Y, Saito D (2016) Verylarge-scale fluctuations in turbulent channel flow at low Reynolds number. Int J Heat Fluid Flow 62:593-597

Miyazaki M (2014) Effect of nano-fibrillated cellulose suspension on transitional two-dimensional channel flow. MSc Thesis. Shinshu University, Japan

Monty J (2004) Developments in smooth wall turbulent duct flows. $\mathrm{Ph} . \mathrm{D}$. Thesis, Univ. Melbourne, Australia

Nishioka M, Asai M (1985) Some observations of the subcritical transition in plane Poiseuille flow. J Fluid Mech 150:441-450

Orszag SA (1971) Accurate solution of the Orr-Sommerfeld stability equation. J Fluid Mech 50:689-703

Orszag SA, Kells LC (1980) Transition to turbulence in plane Poiseuille and plane Couette flow. J Fluid Mech 96:159-205

Patel VC, Head MR (1969) Some observation on skin friction and velocity profiles in fully developed pipe and channel flow. J Fluid Mech 38:181-201

Prandtl L (1933) Neuere Ergebnisse der Turbulenzforschung. Zeitschriftdes Vereins Deutscher Ingenieure 77:105-114. Recent results of turbulence research NACA TM 720
Sano M, Tamai K (2016) A universal transition to turbulence in channel flow. Nat Phys 12:249-253

Schultz MP, Flack KA (2013) Reynolds-number scaling of turbulent channel flow. Phys Fluids 25:025104

Seki D, Matsubara M (2012) Experimental investigation of relaminarizing and transitional channel flows. Phys Fluids 24:1-23

Shimizu M, Manneville P (2019) Bifurcations to turbulence in transitional channel flow. Phys Rev Fluids 4:113903

Tanada Y, Matsubara M (2019) Alternation phenomenon of very large scale feature in turbulent channel flow. In: Annual meeting Japan society fluid mechanics 2019, vol 184 (4 pages in Japanese)

Tillmark N, Alfredsson PH (1992) Experiments on transition in plane Couette flow. J Fluid Mech 235:89-102

Tsukahara T, Seki Y, Kawamura H, Tochio D (2014) DNS of turbulent channel flow at very low Reynolds numbers. 5:1-18. arXiv :1406.0248

Tuckerman LS, Kreilos T, Schrobsdorff H, Schneider TM, Gibson JF (2014) Turbulent-laminar patterns in plane Poiseuille flow. Phys Fluids 26:114103

Watanabe K, Akaoka S, Matsubara M, Kvick M (2012) Statistical properties of disturbances in a transitional channel flow. In: 9th ICFD Sept. 19-21, Sendai, Japan. pp 336-337

Zanoun ES, Nagib H, Durst F (2009) Refined $C_{f}$ relation for turbulent channels and consequences for high-Re experiment. Fluid Dyn Res 41:1-12

Publisher's Note Springer Nature remains neutral with regard to jurisdictional claims in published maps and institutional affiliations. 